\title{
Effects of Multiwalled Carbon Nanotube Reinforced Collagen Scaffolds on the Osteogenic Differentiation of Mesenchymal Stem Cells
}

\author{
Rena Baktur, ${ }^{1}$ Sang-Hee Yoon, ${ }^{2}$ and Soonjo Kwon ${ }^{1,3}$ \\ ${ }^{1}$ Department of Biological Engineering, Utah State University, 4105 Old Main Hill, Logan, UT 84322-4105, USA \\ ${ }^{2}$ Department of Mechanical Engineering, Inha University, 100 Inha-ro, Nam-gu, Incheon 402-751, Republic of Korea \\ ${ }^{3}$ Department of Biological Engineering, Inha University, 100 Inha-ro, Nam-gu, Incheon 402-751, Republic of Korea \\ Correspondence should be addressed to Soonjo Kwon; soonjo.kwon@inha.ac.kr
}

Received 15 October 2013; Accepted 21 October 2013

Academic Editor: Yun Suk Huh

Copyright (C) 2013 Rena Baktur et al. This is an open access article distributed under the Creative Commons Attribution License, which permits unrestricted use, distribution, and reproduction in any medium, provided the original work is properly cited.

\begin{abstract}
With recent advances in nanotechnology, carbon nanotubes (CNTs) have been extensively studied as substrates for cell culture, drug delivery systems, and medical implant materials. However, surprisingly little is known about the effect of CNTs on collective cellular processes (e.g., adhesion, proliferation, and differentiation). This leads to the need for quantitative characterization of the proliferation, differentiation, and mineralization of mesenchymal stem cells (MSCs) on multiwalled CNT-s (MWCNTs-) collagen scaffolds. In here, a set of MWCNTs-collagen scaffolds where three different types of MWCNTs are, respectively, entrapped in reconstituted type I collagen at four different concentrations less than $100 \mathrm{ppm}$ are prepared; the MSC differentiation thereon is investigated by monitoring the transcription factor RunX2 (RunX), transforming growth factor $\beta$ (TGF- $\beta$ ), alkaline phosphatase (AP), osteocalcin, and mineralized nodules of extracellular matrix (ECM). In short, the MWCNT-collagen scaffolds induced significant increases in AP activity and ECM mineralization due to the increased stiffness and strength of the scaffold by entrapping MWCNTs. This offers a potential for controlling MSC differentiation using MWCNT-collagen scaffolds.
\end{abstract}

\section{Background}

There have been significant advances in cell-based therapy and tissue engineering for the repair and replacement of damaged tissues and organs. Now, scientific investigations in this area have been keeping observation upon nanotechnology that has great potential in creating next-generation approaches. More recently, researches have begun to focus on the role of nanostructures and nanomaterials for tissue engineering applications [1]. For example, CNTs introduced by Oberlin et al. in 1976 [2] have been regarded as promising substrates for cell-based therapy and tissue engineering (e.g., cell cultures, drug delivery systems, medical implantable materials, etc.) because of their unique material properties [3-9]. Although nanomaterial-based scaffolds (e.g., CNTs) are believed to provide many critical features of the microenvironments for cell adhesion, proliferation, and differentiation [1], much still remains uncertain and controversial about the effect of the nanomaterial-based scaffolds on the collective cellular processes.

The recent advent of nanotechnology has stimulated an interest in creating a variety of new nanotechnology-based approaches for creating improved scaffolds with engineered tissues. The first pioneering research using composites of synthetic polymers and CNTs has demonstrated great promise that the scaffolds made of the materials can be used for orthopedic tissue engineering applications [10]. The reinforcement of naturally derived polymers (e.g., extracellular matrix (ECM)) with CNTs has been effective to compensate for the inherent limitations of ECM scaffolds such as weak mechanical properties, lack of electrical conductivity, and absence of adhesive and microenvironment-defining moieties [11]. In detail, CNTs have diameters ranging from 0.7 to $2.0 \mathrm{~nm}$ (typically around $1.0 \mathrm{~nm}$ ) and aspect ratios ranging from 100 to 10000 [12], thus playing an important role in fiberreinforced composite materials for scaffolds [13]. Moreover, 
CNTs have a similar viscoelastic property to that observed in soft tissue membranes, so they have been intensively used to increase Young's modulus and tensile strength of hybrid biomaterials $[11,14,15]$.

In this study, MWCNT-reinforced collagen scaffolds were designed to investigate the osteogenic differentiation of MSCs, with the ultimate aim to shed light on the development of multifunctional scaffolds having enhanced mechanical and functional properties for a variety of tissue engineering applications. Our study was spurred by the following questions. (1) How do CNT-based materials affect collective cellular processes of MSCs? (2) Does the addition of CNTs into a naturally derived polymer result in creating a stiffer microenvironment in which osteoprogenitor cells prefer to differentiate into osteocytes? To address the above issues, we prepared a set of MWCNTs-collagen scaffolds where three different types of MWCNTs were entrapped in reconstituted type I collagen at four different concentrations, respectively. The collagen was intended to provide the MSCs with cell binding sites for differentiation, while the MWCNTs were designed to increase the stiffness and tensile strength of the scaffolds. The levels of the proliferation, differentiation, and mineralization of MSCs on the MWCNT-collagen scaffolds were evaluated through measuring osteogenic differentiation markers such as RunX, TGF- $\beta$, AP, osteocalcin, and mineralized nodules of ECM. Our approach is therefore believed to be an exquisite technique for evaluating the effect of scaffolds on MSC differentiation.

\section{Methods}

2.1. Materials and Reagents. Three different types of MWCNTs (HiPCO-derived MWCNT, MWCNT-OH, and MWCNT-COOH) were purchased from Cheap Tubes Inc. (Brattleboro, VT, USA). Rat tail collagen type I was obtained from BD Biosciences (Rockville, MD, USA).The AlamarBlue, Quant-iT PicoGreen dsDNA detection kit, and rat interleukin 6 (IL-6) CytoSet were prepared from Invitrogen Corporation (Carlsbad, CA, USA).

2.2. Proliferation and Differentiation of MSCs. Rat MSCs were obtained from Invitrogen (Carlsbad, CA, USA). The MSCs were cultured in Dulbecco's Modified Eagle's Medium (DMEM) with other supplements: $10 \%$ fetal bovine serum (FBS), $1 \%$ penicillin/streptomycin, and $0.25 \mu \mathrm{g} / \mathrm{mL}$ of amphotericin B. The MSCs were seeded at a density of $4 \times 10^{4}$ cells $/ \mathrm{cm}^{2}$ on the MWCNT-collagen scaffolds in 48-well plates and incubated at $37^{\circ} \mathrm{C}$ and $5 \% \mathrm{CO}_{2}$ in DMEM with other supplements mentioned above. When the MSCs reached confluence, the cell culture media were replaced with differentiating media. The MSCs were differentiated to osteoblastic phenotype by adding $10 \mathrm{mM}$ of $\beta$-glycerophosphate, $50 \mu \mathrm{g} / \mathrm{mL}$ of ascorbic acid, and $10 \mathrm{nM}$ of dexamethasone.

2.3. Preparation of MWCNT-Collagen Scaffolds. Three different types of MWCNTs having a diameter of $20-30 \mathrm{~nm}$ and two different lengths of 10-20 $\mu \mathrm{m}$ (long) and $0.5-2.0 \mu \mathrm{m}$ (short) were added to distilled water and sonicated for
10 minutes with a 10 -second cooling time per minute on ice using a Sonicator 3000 (Misonix, Farmingdale). After sonication, $0.1 \%$ polyvinylpyrrolidone (Fisher Scientific) was added to the MWCNT solution to inhibit the aggregation of the MWCNTs in the solution. Four kinds of concentrations $(10,50,100$, and $855 \mathrm{ppm})$ of MWCNTs were formulated into a fixed concentration $(1 \mathrm{mg} / \mathrm{mL})$ of collagen type I with $10 \% \mathrm{NaHCO}_{3} .1000 \mu \mathrm{L}$ of MWCNT-collagen mixture was transferred to each well plate, followed by incubation for 30 minutes at $37^{\circ} \mathrm{C}$.

2.4. Cell Viability Assay. The viability of MSCs was quantified through the AlamarBlue assay (AB, BioSource International, Camarillo), changing its color from blue and nonfluorescent to red and highly fluorescent under reduction conditions [16]. To examine cell viability, AB was added in an amount equal to $10 \%$ of the MSC culture volume. For each experiment, the fluorescence of $\mathrm{AB}$ was measured from three separate cell monolayers at an excitation wavelength of $545 \mathrm{~nm}$ and an emission wavelength of $590 \mathrm{~nm}$ using a fluorescence microplate reader (Synergy HT Multi-Mode Microplate Reader, Bio-Tek). The cell viability was calculated as the fluorescence intensity ratio of treated cells to untreated cells. As a negative control, $A B$ was loaded to cell culture medium without MSCs. The fluorescence intensity of the plate was measured after 1 hour incubation at $37.0^{\circ} \mathrm{C}$.

2.5. Quantification of Deoxyribonucleic Acid (DNA) Contents. The Quant-iT PicoGreen assay was used to quantify the total amount of DNA present rather than the presence of a specific gene. Briefly, cells were washed once with phosphatebuffered saline (PBS) after removal of culture medium and lysed in $200 \mathrm{~mL}$ solution of $25 \mathrm{mM}$ Tris/0.5\% Triton X-100 ( $\mathrm{pH} 8$ ) followed by three freeze/thaw cycles. Quantification of DNA contents was conducted according the manufacturer's instructions (Molecular Probes). Fluorescence intensity was measured at an excitation wavelength of $480 \mathrm{~nm}$ and an emission wavelength of $520 \mathrm{~nm}$ by a fluorescence microplate reader (Synergy HT Multimode Microplate Reader, Bio-Tek).

2.6. Quantitative Real-Time Polymerase Chain Reaction ( $q R T$ $P C R$ ). Ribonucleic Acid (RNA) was extracted from the samples using the RNeasy Plus Micro Kit (Qiagen) as per manufacturer's protocol with slight modifications. Cell lysate was obtained by crushing the scaffolds while they were submerged in lysis buffer, followed by centrifugation. Because initial RNA concentrations were very low, the process was modified by adding RNase-free water to the scaffold debris and mixing (to dissolve the RNA that had bound to the silica particles from the scaffold) and then centrifuging and adding this supernatant to the cell lysate. The last step of the protocol was modified to elute a total of $20 \mu \mathrm{L}$ of RNA by adding $12 \mu \mathrm{L}$ of RNase-free water twice to a spin column membrane and then centrifuging. qRT-PCR was performed using the RotorGene SYBR Green RT-PCR Kit with a Rotor-Gene qRT-PCR cycler. The genes of interest were glyceraldehyde 3-phosphate dehydrogenase (GAPDH, control), osteocalcin (BGLAP), transcription factor RunX2 (RunX), alkaline phosphatase 
TABLE 1: List of qRT-PCR primers.

\begin{tabular}{|c|c|c|c|}
\hline Gene & Description & Primer & $T_{m}$ \\
\hline \multirow{2}{*}{ GAPDH } & \multirow{2}{*}{ Glyceraldehyde 3-phosphate dehydrogenase } & Forward: 5’ TCGGTGTGAACGGATTTGG 3' & $55.1^{\circ} \mathrm{C}$ \\
\hline & & Reverse: $5^{\prime}$ TCTCCACTTTGCCACTGCA $3^{\prime}$ & $57.4^{\circ} \mathrm{C}$ \\
\hline \multirow{2}{*}{ BGLAP } & \multirow{2}{*}{ Osteocalcin } & Forward: 5' CTGACAAAGCCTTCATGTCCAA 3' & $55.2^{\circ} \mathrm{C}$ \\
\hline & & Reverse: 5' GCCGGAGTCTGTTCACTACCTT 3' & $58.3^{\circ} \mathrm{C}$ \\
\hline \multirow{2}{*}{ RunX } & \multirow{2}{*}{ Transcription factor RunX2 } & Forward: 5' CTTCAAGGTTGTAGCCCTCGGA 3' & $58.4^{\circ} \mathrm{C}$ \\
\hline & & Reverse: $5^{\prime}$ ATGACGGTAACCACAGTCCCA $3^{\prime}$ & $58.5^{\circ} \mathrm{C}$ \\
\hline \multirow{2}{*}{$\mathrm{AP}$} & \multirow{2}{*}{ Alkaline phosphatase } & Forward: 5’ AAGGATATCGACGTGATCATGG 3' & $54.1^{\circ} \mathrm{C}$ \\
\hline & & Reverse: 5' GGCCTTCTCATCCAGTTCGTA 3' & $56.7^{\circ} \mathrm{C}$ \\
\hline \multirow{2}{*}{ Smad4 } & \multirow{2}{*}{ SMAD family member 4} & Forward: 5' CACAAGTCAGCCGGCCAGTATT 3' & $59.4^{\circ} \mathrm{C}$ \\
\hline & & Reverse: $5^{\prime}$ TTCCAGTCCAGGTGGTAGT $3^{\prime}$ & $59.0^{\circ} \mathrm{C}$ \\
\hline
\end{tabular}

(AP), and SMAD family member 4 (SMAD4) (Table 1). The primers used in this study are listed in Table 1. Analysis of the cycle data was assessed using the double $\Delta C t$ method with a threshold value of $20 \%$ above the background. Efficiency values were empirically derived from 3 plates of differentiated cells (grown in the same media as a positive control) for the purposes of this analysis. Fold changes were determined through Ct value comparison to the negative control. All data was subject to normalization to the value of a housekeeping gene (GAPDH) for each experimental condition and specimen. All data was analyzed by analysis of variance (ANOVA) technique.

2.7. Alkaline Phosphatase Activity. The AP detection was detected by calf intestinal alkaline phosphatase (CIAP, Promega) to measured cell differentiation. For AP detection, a substrate $(0.4 \mathrm{mM}$ of CSPD with Sapphire II, CD100RX, Applied Biosystems) was used. A preparation method of cell lysates was the same as a quantification method of DNA contents. Chemiluminescence of AP activity in each sample was measured by a chemiluminescence microplate reader (Synergy HT Multimode Microplate Reader, Bio-Tek). The light output from each sample was integrated for 10 seconds after a delay of 2 seconds. The chemiluminescence data were calibrated with a CIAP standard curve to present AP activity. All AP activity data were normalized by DNA contents.

2.8. Von Kossa Staining. The mineralized nodules on MSC layers were quantified by the Von Kossa stain method. The cell layers were washed twice with PBS, fixed in phosphate buffered formalin for $10 \mathrm{~min}$, washed once with deionized (DI) water, and serially dehydrated in $70 \%, 95 \%$, and $100 \%$ ethanol two times and dried. The cell layers were then rehydrated in 100\%, 95\%, and $80 \%$ ethanol and DI water sequentially. After addition of $5 \%$ silver nitrate solution, the cell layers were exposed to sunlight for 20 minutes after which the plate was rinsed with DI water. After addition of sodium thiosulfate (5\%) for 3 minutes, the cell layers were then rinsed in DI water. Acid fuchsin counterstain $(5 \mathrm{~mL}$ of $1 \%$ acid fuchsin $+95 \mathrm{~mL}$ of picric acid $+0.25 \mathrm{~mL}$ of $12 \mathrm{~N}$ hydrochloric acid) was added to the cell layers for 5 minutes. The cells were washed with DI water, twice with 95\% ethanol, twice with 100\% ethanol, and dried for image analysis.
2.9. Measurement of Mechanical and Surface Property of MWCNT-Collagen Scaffolds. The surface hydrophobicity of the MWCNT-collagen scaffolds was determined from measuring static contact angles. A $2 \mu \mathrm{L}$ water droplet was dripped on the dried surface of the MWCNT-collagen scaffolds. The image was captured immediately after inoculation. Contact angles were measured by a VCA-Optima digital contact angle instrument (VCA Optima, AST Products Inc.). The contact angle data were obtained by averaging those of four droplets. Compressive testing was performed on the MWCNT-collagen scaffolds located on a 24 -well plate. The height of each sample, from the bottom of the well plate, was approximately $5 \mathrm{~mm}$ and the surface area was $1.9 \mathrm{~cm}^{2}$, whereas the diameter of the probe was $10 \mathrm{~mm}$. The test was performed at extension rate of $10 \mathrm{~mm} / \mathrm{min}$ until total extension of $2 \mathrm{~mm}$ using an Instron 5542 apparatus (Instron, Norwood). Young modulus was determined from the elastic regime slope of stress-strain curves ( $<40 \%$ strain).

2.10. Statistical Analysis. Statistically significant difference was set at $P \leq 0.05$. Statistical analyses were carried out using one-way ANOVA followed by Dunnett's multiple comparison test.

\section{Results}

3.1. Messenger RNA (mRNA) Expression for RunX, SMAD4, $A P$, and BGLAP. The mRNA expression of markers associated with osteogenesis in MSCs was analyzed 3 weeks after the replacement of differentiating media. The mRNA level of markers used in this study included RunX, SMAD4, AP, and BGLAP (osteocalcin). The data was presented as a fold change over the negative control of proliferating MSCs on MWCNT-collagen scaffolds having different concentrations of MWCNTs (Figure 1). The mRNA level of AP on MWCNTcollagen having high concentrations of MWCNTs (50 and $100 \mathrm{ppm}$ ) was significantly increasing, compared to that of the collagen scaffold without MWCNTs (i.e., collagen only). The mRNA levels of the other three markers were not significantly changed.

3.2. Effects of MWCNT-Collagen Scaffolds with Varying MWCNT Concentrations on MSC Differentiation. Different 


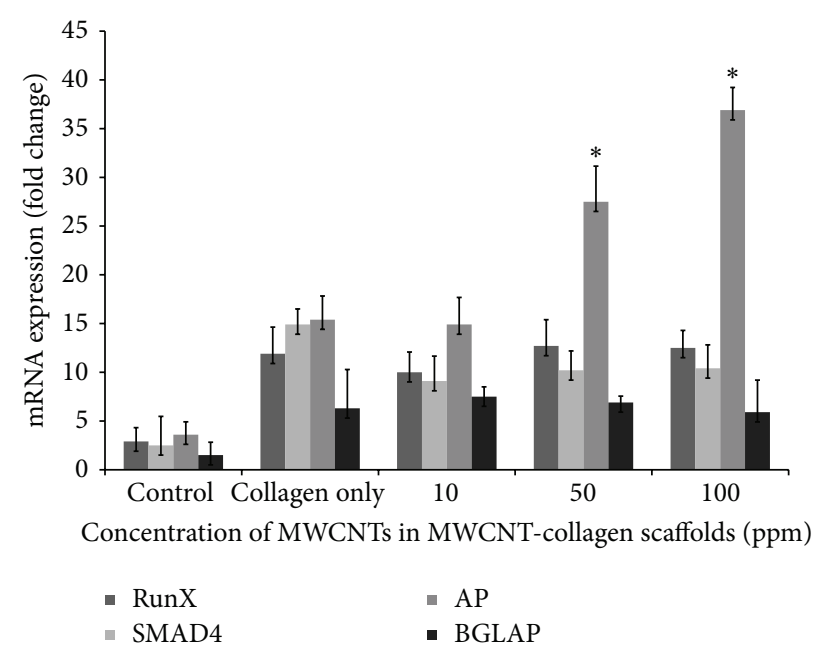

FIGURE 1: qRT-PCR analysis. Data are analyzed by the double $\Delta C t$ method using the GAPDH as a control gene and the BIO specimens (cells grown on bioactive glass in standard proliferation media) as the reference sample. All data are presented as upregulation over the reference specimen, corrected for GAPDH expression. * denotes a significantly higher difference than the control (day 0$)(P<0.05)$.

concentrations of MWCNTs were added to the collagen scaffolds to prepare the MWCNT-collagen scaffolds. MSCs were seeded onto the MWCNT-collagen scaffolds. When MSCs reached confluence, the cell culture media were replaced with differentiating media. AP activity was measured at four-day intervals. A collagen itself enhanced MSCs differentiation; the addition of the MWCNTs to collagen scaffolds induced an additional increase in MSC differentiation. In detail, MWCNT-collagen scaffolds induced significantly higher level of AP activity than the "control" (i.e., treated plastic surfaces for cell culture) 12 days after replacement with differentiating media. There was no significant difference in AP activity of MSCs on scaffolds with different concentrations of MWCNTs between 10 and $100 \mathrm{ppm}$, showing that MWCNT-collagen scaffold-induced AP activity did not have dose dependency (Figure 2). Although AP levels were higher in the MWCNT-collagen scaffolds at all MWCNT concentrations, the transparency of the scaffolds was reduced as the concentrations of MWCNTs increased (Figure 2). The transparency of the MWCNT-collagen scaffolds was appropriate for microscopic observation at lower MWCNT concentrations $(<100 \mathrm{ppm})$ but was significantly reduced at higher MWCNT concentrations ( $>100 \mathrm{ppm})$. In terms of transparency and AP activity, the MWCNT-collagen scaffolds having $10 \mathrm{ppm}$ MWCNT were believed to be the optimal ones.

3.3. Effects of MWCNT Type on MSC Differentiation. Three different types of MWCNTs (i.e., HiPCO-derived MWCNT, MWCNT-OH, and MWCNT-COOH) having two different lengths (i.e., short and long) were added to the collagen scaffolds at a concentration of $10 \mathrm{ppm}$. When MSCs reached confluence, the cell culture media were replaced with differentiating media. AP activity was measured at four-day

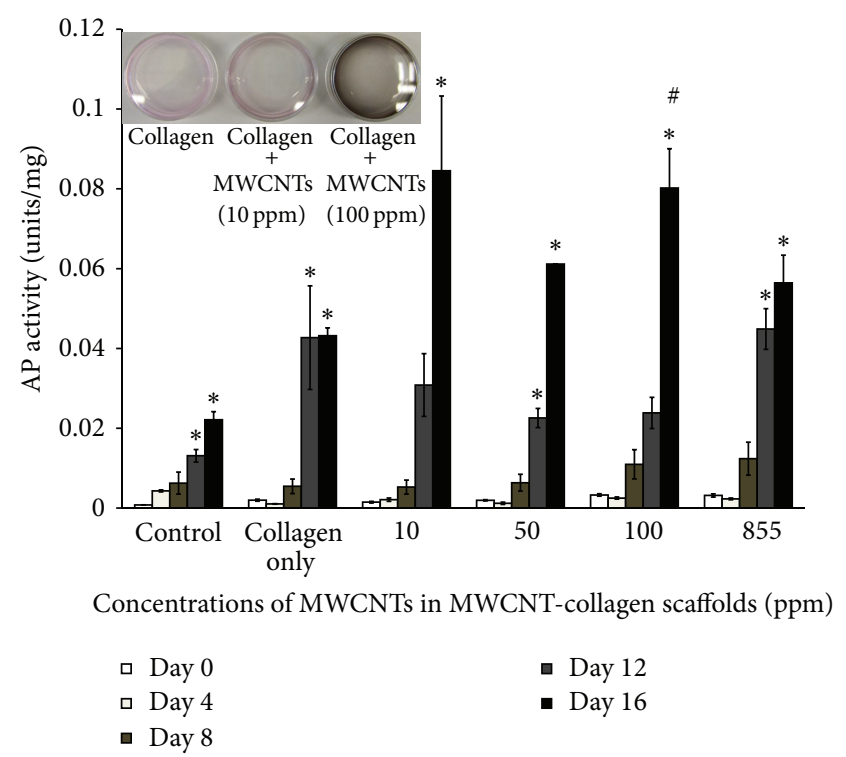

FIGURE 2: Effects of the MWCNT-collagen scaffolds with different MWCNT concentrations on MSC differentiation. AP activity is measured at four-day intervals from the differentiating media in which MSCs are differentiated on the MWCNT-collagen scaffolds with different MWCNT concentrations. * denotes a significantly higher difference than the control $(0$ hour $)(P<0.05)$ and \# denotes a significantly higher difference than both the control group and collagen only group at the same time point $(P<0.05)$. "Control" represents the surface of a regular cell culture-treated dish. All AP activity data are normalized by DNA contents.

intervals. The level of AP activity was higher on all different types of MWCNT-collagen scaffolds than on two groups (i.e., control and collagen only). At day 12, all types of MWCNT-collagen scaffolds induced higher levels of MSC differentiation compared to the control and collagen only $(P<0.05)$ (Figure 3). However, different types of MWCNTs did not show significant differences in AP activity.

\subsection{Effects of MWCNT Type on the Formation of Mineralized} Matrix Nodules. The dependency of the formation of mineralized matrix nodules on MWCNT type entrapped in collagen was also verified. All experiments for this purpose were made at the same conditions as the previous section (effects of MWCNT type on MSCs differentiation). As the days increased, MSCs induced the increased mineralized nodules. The area of mineralized matrix nodules was significantly higher at day 15 (Figure 3). MSCs on both MWCNT-collagen and MWCNT-OH-collagen scaffolds with short length (0.5$2.0 \mu \mathrm{m})$ induced significant increase in mineralization of matrix nodules (Figure 4).

\subsection{Effects of MWCNTs on Cell Viability and Cell Proliferation.} There was no significant change in cell viability among different types of MWCNT-collagen scaffolds although significant decrease in cell viability on MWCNT-collagen scaffolds was observed at day 4 (Figure 5(a)). All types of MWCNTcollagen scaffolds did not induce a detrimental effect on cell 


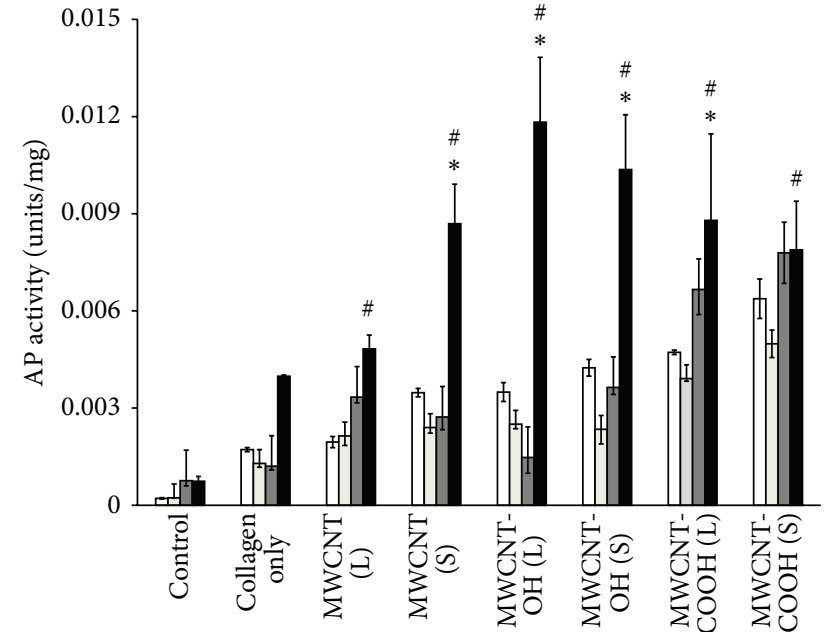

Different types of MWCNT-collagen scaffolds

$$
\begin{array}{ll}
\square \text { Day } 0 & \square \text { Day } 8 \\
\square \text { Day } 4 & \text { - Day } 12
\end{array}
$$

Figure 3: Effects of MWCNT types on MSC differentiation. * denotes a significantly higher difference than the control (0 hour) $(P<0.05)$. \# denotes a significantly higher difference than the control and collagen only at the same time point $(P<0.05)$. MWCNT (L) and MWCNT-OH (S) mean MWCNT-collagen scaffold (long) and MWCNT-OH-collagen scaffold (short), respectively.

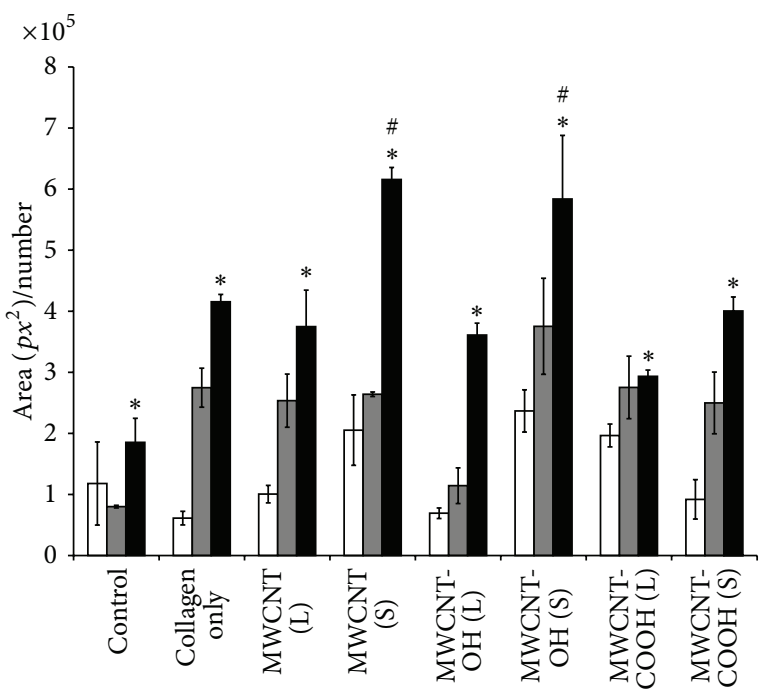

Different types of MWCNT-collagen scaffolds

$\square$ Day 5

- Day 10

- Day 15

FIGURE 4: Effects of MWCNT types on the formation of mineralized matrix nodules. Mineralized nodule formation of MSC monolayers is analyzed at five-day intervals on MWCNT-collagen scaffold having a MWCNT concentration of $10 \mathrm{ppm} . *$ denotes a significantly higher difference than the control (day 5) $(P<0.05)$ and \# denotes a significantly higher difference than the control at the same time point $(P<0.05)$. proliferation (Figure 5(b)). In most cases, cell proliferation increased during this period.

3.6. Mechanical Properties of MWCNT-Collagen Scaffolds. Contact angles of collagen scaffolds were higher than that of the control, but the difference was not significant. In addition, there was also no significant difference in contact angles among different types of MWCNT-collagen scaffolds (Figure 6). The elastic modulus and stiffness, however, increased as the MWCNT concentration in collagen scaffolds increased. Different types of MWCNTs at the same concentration did not affect the changes in stiffness of MWCNT-scaffolds (data not shown here). Ten to $100 \mathrm{ppm}$ of MWCNTs in the MWCNT-collagen scaffolds induced significantly higher stiffness than others (Figure 7).

\section{Discussion}

Cell functions in tissue are influenced by the microenvironment that is generated locally at micro- or nanoscale level by cell-cell or cell-soluble factors or cell-ECM interactions. Each cell is specifically modulated by the microenvironments in which it resides. For example, brain tissues on a highly compliant matrix are softer than bone tissues on a highly noncompliant one. Consequently, neural cell growth, survival, and differentiation are favored by highly compliant matrices whereas osteoblast differentiation and survival occur preferentially on stiffer extracellular matrices whose material properties are very similar to a newly formed bone [17]. ECM or ECM-like structures can create cellular microenvironment for specific cells or tissues. Although ECM scaffolds yield good cellular microenvironment, the scaffolds are known to be reinforced by some stiff nanomaterials to overcome their inherent limitations (e.g., weak mechanical properties, lack of electrical conductivity, and absence of microenvironmentdefining moieties) [11].

We hypothesized that augmenting the properties of naturally derived polymers (collagen here) through incorporation of MWCNTs might enhance in vitro osteogenic differentiation and mineralization of MSCs. In this study a set of MWCNTs-collagen scaffolds, in which three different types of MWCNTs were entrapped in reconstituted type I collagen at four different concentrations, were fabricated. The proliferation, differentiation, mineralization, and cell viability of MSCs on the MWCNT-collagen scaffolds were evaluated as a function of the entrapped amount of MWCNTs. Although MWCNTs were strongly entrapped in collagen $(1 \mathrm{mg} / \mathrm{mL})$ at high concentrations (below $100 \mathrm{ppm}$ ), the MWCNT-collagen scaffolds showed excellent transparency, which is essential to cell characterization through microscopic observation (Figure 2). In here, MSCs were used to mimic the subsequent stages of osteoblastic differentiation in vitro and the MSCs were observed to mature into osteoblasts after cell culture media were exchanged for differentiating media. During this process, AP activity (Figures 1, 2, and 3) increased. Subsequently, cells started to mature the extracellular matrix and mineralized matrix nodules appeared (Figure 4). Higher level of AP activity was observed from the MSCs on 


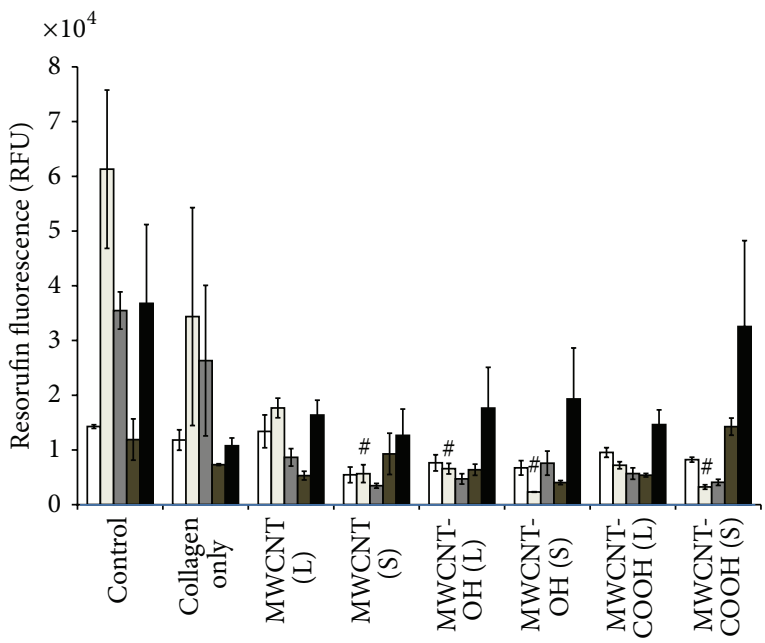

Different types of MWCNT-collagen scaffolds
- Day 12

- Day 16

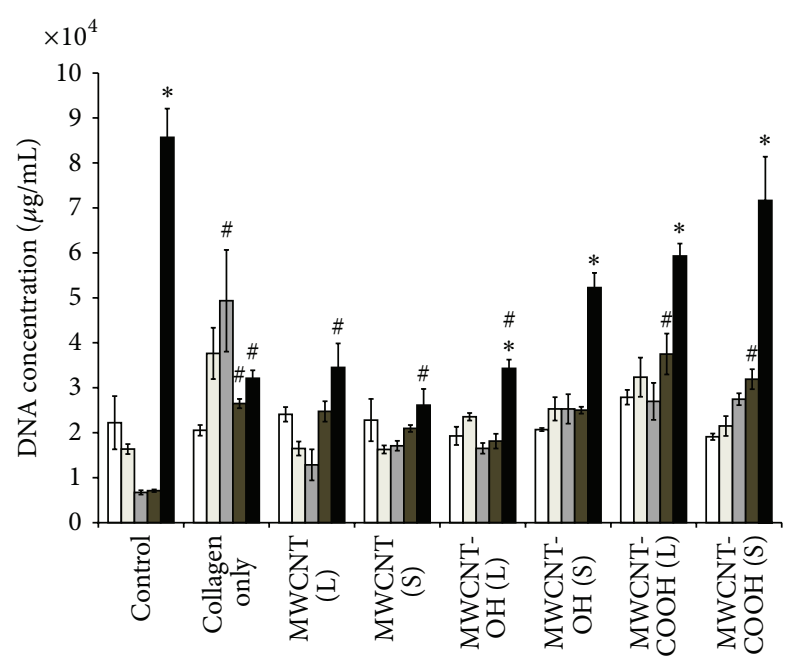

Different types of MWCNT-collagen scaffolds
- Day 12

- Day 16

(a)

(b)

FIGURE 5: Effects of MWCNTs on cell viability and cell proliferation. (a) Cell viability and (b) cell proliferation are measured after media replacement with differentiating media. $*$ denotes a significantly higher difference than the control (day 0$)(P<0.05)$. \# denotes a significantly higher difference than the control at the same time point $(P<0.05)$.

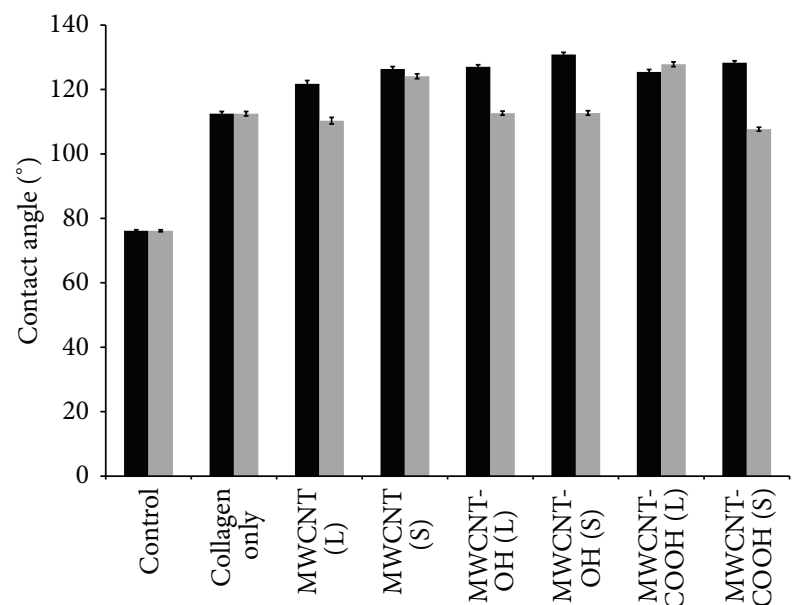

Different types of MWCNT-collagen scaffolds

- $10 \mathrm{ppm}$

- $100 \mathrm{ppm}$

Figure 6: Surface properties of the MWCNT-collagen scaffolds. Surface hydrophobicity of the MWCNT-collagen scaffolds is determined by measuring the static contact angle of a water droplet of $2 \mu \mathrm{L}$. Each contact angle data is the average of four droplets.

MWCNT-collagen scaffolds and collagen in comparison to the cells on the control (Figures 1 and 2). Different types of MWCNTs did not make significant differences in AP activity on MWCNT-collagen scaffolds (Figure 3). However, MSCs

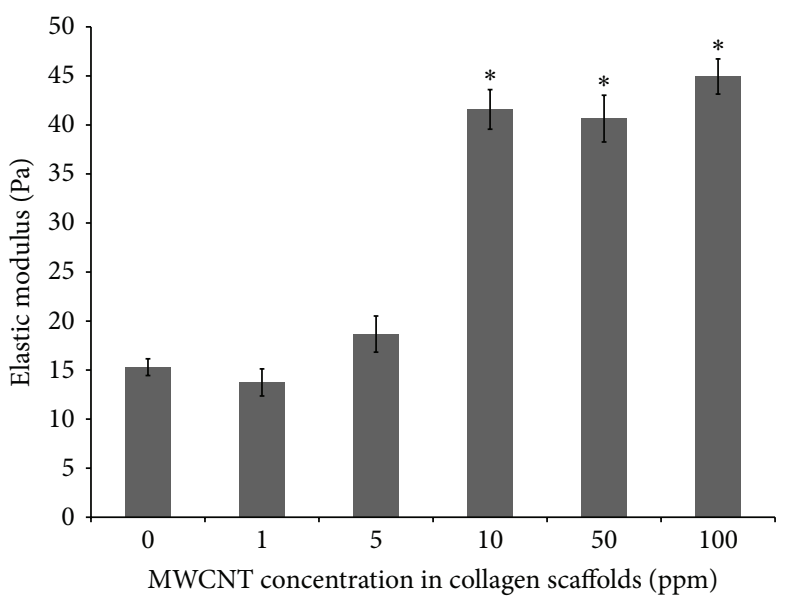

FIGURE 7: Measurements of elastic modulus in the collagen scaffolds with different MWCNT concentrations. Compressive testing is performed on the MWCNT-collagen scaffolds located on a 24-well plate. Young modulus is determined from the elastic regime slope of stress-strain curves ( $<40 \%$ strain). $*$ denotes a significant difference in elastic modulus from the collagen only group.

on both MWCNT-collagen and MWCNT-OH-collagen scaffolds with short length $(0.5-2.0 \mu \mathrm{m})$ induced significant increase in mineralization of matrix nodules (Figure 4).

In terms of structure, collagen provides tensile strength to tissues via its hierarchical assembly of subunits. In addition to biologic signaling and macroscopic mechanical properties, collagen also possesses nanoscale features that 
are mediators of cell activity. Previous studies have shown that the changes made by certain features (e.g., structural curvature of collagen fibrils) can regulate the activities of adherent cells. Nanostructures and nanoscale materials play important roles in tissue engineering applications. As a strong candidate, CNTs have been used to modulate cell behavior through mechanically reinforcing or tailoring the structural properties of tissue engineered scaffolds. Furthermore, CNTs have been used to increase the surface roughness and surface area of scaffolds for cell adhesion [1]. In this study, collagen itself enhanced MSCs differentiation and mineralization due to its nanoscale features. That is, the presence of MWCNTs in the collagen scaffolds yielded a further increase in MSC differentiation and mineralization. This additional increase in MSC differentiation and mineralization is thought to be due to a heightened stiffness and tensile strength of the MWCNT-collagen scaffolds (Figure 7). Although no significant difference in contact angles among the MWCNTreinforced collagen scaffolds is observed (Figure 6), it is still not clear that the surface properties (e.g., topography) of MWCNT-collagen scaffolds affect MSC differentiation and mineralization in this study. Moreover, although the effect of the functional groups $(-\mathrm{OH}$ or $-\mathrm{COOH})$ attached to both ends of MWCNTs was partially verified in this study, full analysis (using different methods) for other functionalized MWCNTs (e.g., $-\mathrm{NH}_{2}$ ) will be required to determine whether surface properties of nanocomposite scaffolds increase cell differentiation. In a way, the results obtained from here give us a hint for understanding the inflammatory response of MSCs because in vivo MSCs are known to migrate to the sites of inflammation. They engraft in a variety of tissues and appear to play an active role in tissue repair and regeneration by secreting chemokines as well as differentiating into mature cell types [18-20]. This differentiation is likely to be initiated and directed as soon as the MSCs sense the changes in their local microenvironment including mechanical properties. MSCs can also produce matrix metalloprotease (MMP) during cell proliferation and differentiation, which may affect the mechanical properties of the scaffolds. Despite the secretion of active forms of MMPs by MSCs, MMP enzyme activity is not detected in MSC-conditioned medium (MSC-CM) due to tissue inhibitors of metalloproteinases- (TIMPs-) mediated inhibition [21]. Thus, the degradation rate of the scaffold in this study is thought to be very low and also the degradation effect of the scaffold on cell proliferation, differentiation, and mineralization might be negligible.

Ongoing work is focusing on incorporating nanoscale materials into the inside or outer surfaces of threedimensional (3D) scaffolds to gather an in-depth understanding of the dependence of collective cellular processes on the mechanical stiffness of the scaffolds. Further studies will be also made to characterize how the mechanical property changes trigger MSC differentiation to a specific lineage in complex microenvironments where inducers for multiple cell types are present. The findings of this study, together with ongoing work, will lead to better understanding of cellular biomechanics in MSC proliferation and differentiation that is a critical element for the development of functional tissues in vitro.

\section{Acknowledgments}

Funding for this study was provided by Vice President for Research (VPR) in the Utah State University and Inha University Research Grant (INHA-47289-01).

\section{References}

[1] B. M. Baker, A. M. Handorf, L. C. Ionescu, W.-J. Li, and R. L. Mauck, "New directions in nanofibrous scaffolds for soft tissue engineering and regeneration," Expert Review of Medical Devices, vol. 6, no. 5, pp. 515-532, 2009.

[2] A. Oberlin, M. Endo, and T. Koyama, "Filamentous growth of carbon through benzene decomposition," Journal of Crystal Growth, vol. 32, no. 3, pp. 335-349, 1976.

[3] S. Iijima, "Helical microtubules of graphitic carbon," Nature, vol. 354, no. 6348, pp. 56-58, 1991.

[4] D. A. Heller, S. Baik, T. E. Eurell, and M. S. Strano, "Singlewalled carbon nanotube spectroscopy in live cells: towards long-term labels and optical sensors," Advanced Materials, vol. 17, no. 23, pp. 2793-2799, 2005.

[5] L. Z. Gao, L. Nie, T. H. Wang et al., "Carbon nanotube delivery of the GFP gene into mammalian cells," ChemBioChem, vol. 7, no. 2, pp. 239-242, 2006.

[6] D. Cui, F. Tian, Y. Kong, I. Titushikin, and H. Gao, "Effects of single-walled carbon nanotubes on the polymerase chain reaction," Nanotechnology, vol. 15, no. 1, pp. 154-157, 2004.

[7] R. L. Price, M. C. Waid, K. M. Haberstroh, and T. J. Webster, "Selective bone cell adhesion on formulations containing carbon nanofibers," Biomaterials, vol. 24, no. 11, pp. 1877-1887, 2003.

[8] H. Hu, Y. Ni, V. Montana, R. C. Haddon, and V. Parpura, "Chemically functionalized carbon nanotubes as substrates for neuronal growth," Nano Letters, vol. 4, no. 3, pp. 507-511, 2004.

[9] E. Jan and N. A. Kotov, "Successful differentiation of mouse neural stem cells on layer-by-layer assembled single-walled carbon nanotube composite," Nano Letters, vol. 7, no. 5, pp. 11231128, 2007.

[10] P. R. Supronowicz, P. M. Ajayan, K. R. Ullmann, B. P. Arulanandam, D. W. Metzger, and R. Bizios, "Novel current-conducting composite substrates for exposing osteoblasts to alternating current stimulation," Journal of Biomedical Materials Research, vol. 59, no. 3, pp. 499-506, 2002.

[11] T. Dvir, B. P. Timko, D. S. Kohane, and R. Langer, "Nanotechnological strategies for engineering complex tissues," Nature Nanotechnology, vol. 6, no. 1, pp. 13-22, 2011.

[12] P. M. Ajayan, J.-C. Charlier, and A. G. Rinzler, "Carbon nanotubes: from macromolecules to nanotechnology," Proceedings of the National Academy of Sciences of the United States of America, vol. 96, no. 25, pp. 14199-14200, 1999.

[13] R. A. MacDonald, B. F. Laurenzi, G. Viswanathan, P. M. Ajayan, and J. P. Stegemann, "Collagen-carbon nanotube composite materials as scaffolds in tissue engineering," Journal of Biomedical Materials Research A, vol. 74, no. 3, pp. 489-496, 2005.

[14] J. Suhr, P. Victor, L. Ci et al., "Fatigue resistance of aligned carbon nanotube arrays under cyclic compression," Nature Nanotechnology, vol. 2, no. 7, pp. 417-421, 2007.

[15] S.-F. Wang, L. Shen, W.-D. Zhang, and Y.-J. Tong, "Preparation and mechanical properties of chitosan/carbon nanotubes composites," Biomacromolecules, vol. 6, no. 6, pp. 3067-3072, 2005. 
[16] H. Gloeckner, T. Jonuleit, and H.-D. Lemke, "Monitoring of cell viability and cell growth in a hollow-fiber bioreactor by use of the dye Alamar Blue," Journal of Immunological Methods, vol. 252, no. 1-2, pp. 131-138, 2001.

[17] D. T. Butcher, T. Alliston, and V. M. Weaver, "A tense situation: forcing tumour progression," Nature Reviews Cancer, vol. 9, no. 2, pp. 108-122, 2009.

[18] A. I. Caplan, "Adult mesenchymal stem cells for tissue engineering versus regenerative medicine," Journal of Cellular Physiology, vol. 213, no. 2, pp. 341-347, 2007.

[19] A. Arthur, A. Zannettino, and S. Gronthos, "The therapeutic applications of multipotential mesenchymal/stromal stem cells in skeletal tissue repair," Journal of Cellular Physiology, vol. 218, no. 2, pp. 237-245, 2009.

[20] V. F. M. Segers, I. van Riet, L. J. Andries et al., "Mesenchymal stem cell adhesion to cardiac microvascular endothelium: activators and mechanisms," The American Journal of Physiology: Heart and Circulatory Physiology, vol. 290, no. 4, pp. H1370H1377, 2006.

[21] T. P. Lozito and R. S. Tuan, "Mesenchymal stem cells inhibit both endogenous and exogenous MMPs via secreted TIMPs," Journal of Cellular Physiology, vol. 226, no. 2, pp. 385-396, 2011. 

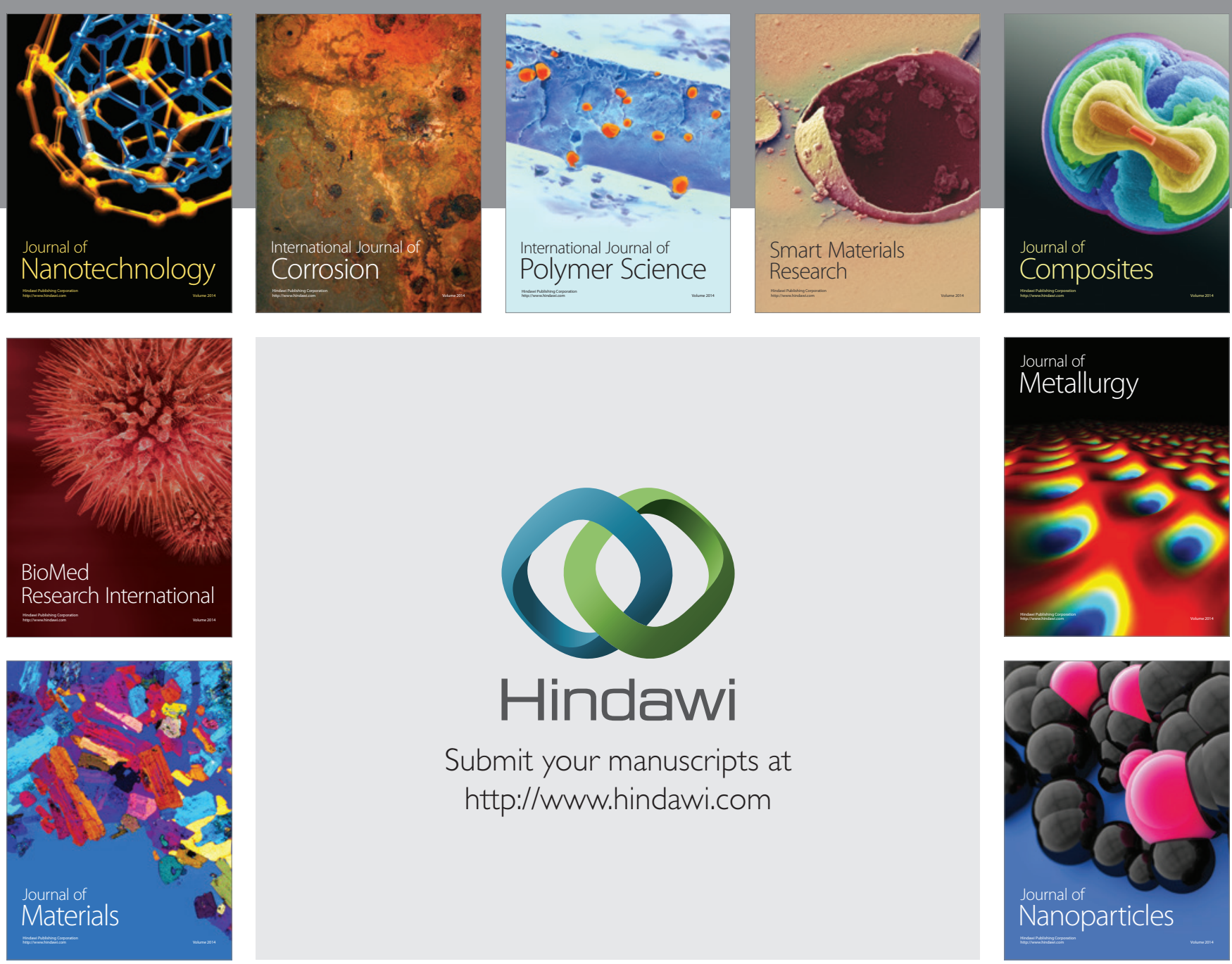

Submit your manuscripts at http://www.hindawi.com
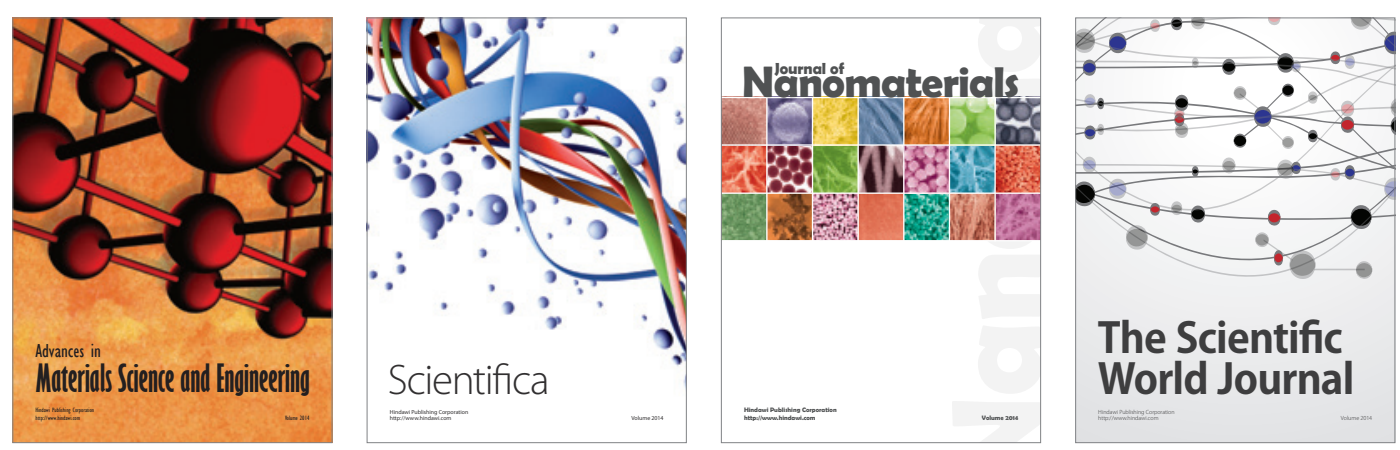

\section{The Scientific World Journal}
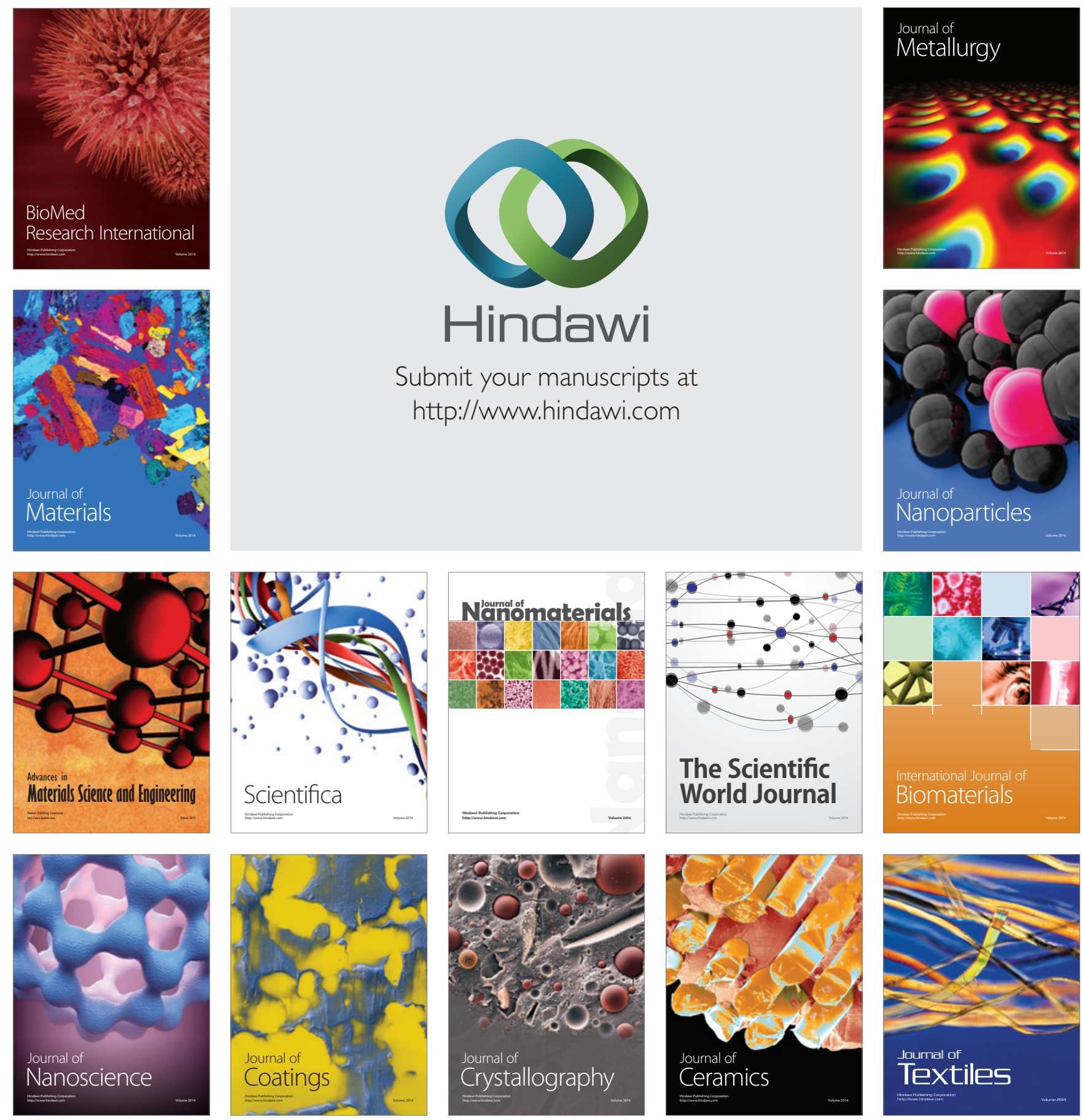